\title{
A general theorem on the divergence of vortex beams
}

\author{
Giuseppe Vallone,, , Giuseppe Parisi, ${ }^{2}$ Fabio Spinello, ${ }^{1,2}$ Elettra Mari, ${ }^{2}$ Fabrizio Tamburini,${ }^{2}$ and Paolo Villoresi ${ }^{1}$ \\ ${ }^{1}$ Department of Information Engineering, University of Padova, via Gradenigo 6/B, 35131 Padova, Italy \\ ${ }^{2}$ Twist-Off s.r.l., via della Croce Rossa 112, I-35129 Padova, Italy
}

\begin{abstract}
The propagation and divergence properties of beams carrying orbital angular momentum (OAM) play a crucial role in many applications. Here we present a general study on the divergence of optical beams with OAM. We show that the mean absolute value of the OAM imposes a lower bound on the value of the beam divergence. We discuss our results for two different definitions of the divergence, the so called rms or encircled-energy. The bound on the rms divergence can be expressed as a generalized uncertainty principle, with applications in long-range communication, microscopy and $2 \mathrm{D}$ quantum systems.
\end{abstract}

\section{INTRODUCTION}

Since the initial work by Allen et al. 11, the orbital angular momentum (OAM) of light has attracted increasing interest in multiple fields, including microscopy [2], optical trapping [3], astronomy [4, 5, radio [6] and optical communication [7-10] and fundamental physics [11-13]. OAM beams are characterized by a singular phase factor $\exp (i \ell \phi)$, where $\phi$ refers to the azimuthal angle around the beam axis and the topological charge, $\ell$, is an integer parameter representing different OAM values [1]. When $\ell \neq 0$, the beam presents an optical vortex on its axis due to the phase singularity.

The study of the propagation and divergence properties of OAM beams play a crucial role in many applications, and in particular in long-range communication systems [14, 15] and microscopy. In this context, standard Laguerre-Gaussian (LG) beams have already been studied in detail 16-20. Recently 21, a preliminary analysis was also carried out on the Circular Beam $(\mathrm{CiB})$, which represents a general analytical solution of the paraxial wave equation with OAM 21, 22. Indeed, several well known beams carrying OAM - such as the standard 23 . or elegant 24. LG beams, Bessel-Gauss beams [25, optical vortex beams [26] and others 222 - are particular cases of CiBs obtained by setting specific values to the beams' parameters. Moreover, CiBs naturally arise when $q$-plates [27] or phase plates [28 are used to general OAM from a Gaussian beam [21, 29].

The divergence properties of generic incoherent superposition of LG modes can be easily bounded by knowing the divergence angles of the Laguerre-Gauss beams. However, for generic (coherent) beams - that can be always expressed as a coherent superposition of LG modes - there are no known bounds. One could image that by coherently adding different LG modes with the same OAM it could be possible to lower the divergence of the beam, by keeping fixed the value of the OAM. A central question is thus the following: by fixing the OAM content and arbitrarily changing the radial profile of a beam is it possible to reduce its divergence? The above question

\footnotetext{
* vallone@dei.unipd.it
}

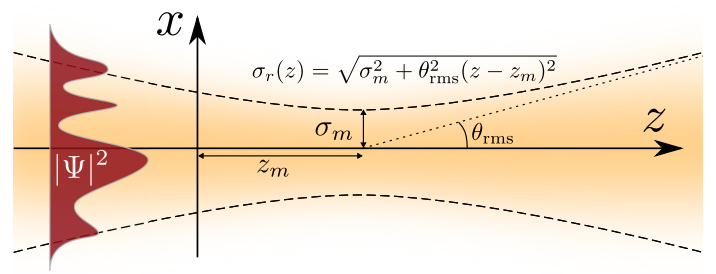

FIG. 1. (Color online) Pictorial representation of the rms divergence $\theta_{\mathrm{rms}}$ of a generic paraxial beam $\Psi(x, y, z)$ propagating along the $z$ axis. Dashed lines represent $\sigma_{r}(z)$. The parameters $\sigma_{m}$ and $z_{m}$ are the minimum value of $\sigma_{r}(z)$ and its location respectively.

is crucial for applications that require the optimization of the far-field propagation or the focusing properties of the used beam.

Here we answer to the above question by presenting a general study on the propagation and divergence properties of OAM beams, including CiBs as special case. The study on the divergence of optical beams will led to a formulation of an uncertainty principle, based on the mean value of the OAM. Our main result can be stated as follows: for any optical monochromatic paraxial beam with a mean value of OAM given by $\langle|\ell|\rangle$, the product of the spatial extent, $\sigma_{r}$, and the spread $\sigma_{k}$ in the wavectorspace is lower bounded by

$$
\sigma_{k} \sigma_{r} \geq 1+\langle|\ell|\rangle
$$

This result can be heuristically explained by noticing that, for an optical beam with $\ell$ units of OAM, the Poynting vector angle with respect to the propagation axis is given by $\ell /(k r)$ [30, 31]. Then, the "spreading" of the Poynting vectors, related to the beam divergence, tends to increase with $\ell$. Our result is a kind of no-go theorem: any optimization of the beam radial profile cannot improve the divergence below the limit imposed by eq. (1).

We now derive eq. (1) and show how the inequality could be also exploited in microscopy and formulated as a position-momentum uncertainty principle. 


\section{BOUNDING THE RMS DIVERGENCE}

Let's consider a generic monochromatic and paraxial beam propagating in the $z$ direction: its wavefuction can be written as $\Psi(x, y, z) e^{i \omega t}$, where $\omega$ is the angular frequency and $k=\omega / c$ the wavenumber. By defining $\mathbf{x}_{\perp}=(x, y)=(r, \phi)$ as the coordinate on the plane transverse to propagation, without loss of generality we can consider only beams with $\left\langle\mathbf{x}_{\perp}(z)\right\rangle=0$ [32. The starting point for our analysis is the so called root-mean-square (rms) far field angle of divergence $\theta_{\text {rms }}$. This is defined, for a generic paraxial beam $\Psi$, as

$$
\theta_{\mathrm{rms}}=\lim _{z \rightarrow \infty} \frac{\sigma_{r}(z)}{z}
$$

where $\sigma_{r}^{2}(z)$ is the variance of the intensity $I(r, \phi, z)=$ $|\Psi(r, \phi, z)|^{2}$ given by:

$$
\sigma_{r}^{2}(z)=\int \mathrm{d} \phi \int \mathrm{d} r r^{3} I(r, \phi, z) .
$$

The value of $\sigma_{r}(z)$ represents the beam radius at location $z$ [16, 33 34. As underlined by the r.h.s. of eq. (2), the far field angle of divergence $\theta_{\text {rms }}$ quantifies the asymptotic rate of variation of $\sigma_{r}(z)$.

The divergence $\theta_{\text {rms }}$ can be explicitly evaluated by exploiting the expansion of $\Psi$ in LG modes, a complete (orthonormal) basis set of solutions of the paraxial wave equation. Due to the completeness of the LG basis, any paraxial beam $\Psi$ can be expanded as:

$$
\Psi(r, \phi, z)=\sum_{n=0}^{+\infty} \sum_{\ell=-\infty}^{+\infty} \psi_{n, \ell} \mathrm{LG}_{n, \ell}(r, \phi, z) .
$$

In the above expression the (complex) expansion coefficients $\psi_{n, \ell}$ are adimensional and they are normalized such that $\sum_{n, \ell}\left|\psi_{n, \ell}\right|^{2}=1$. The waist parameter $w_{0}$ of the LG modes determines the physical size of $\Psi$ (see appendix A). The integers $n$ and $\ell$ respectively represent the radial quantum number and the OAM content of each LG mode.

As first derived by Siegman in [17, the square of the beam radius $\sigma_{r}^{2}(z)$ of a generic beam has a parabolic dependence on $z$, namely $\sigma_{r}^{2}(z)=\sigma_{m}^{2}+\theta_{\text {rms }}^{2}\left(z-z_{m}\right)^{2}$. The parameters $\sigma_{m}$ and $z_{m}$ represent the minimum value of $\sigma_{r}(z)$ and its location on the $z$-axis respectively. A pictorial representation of $\sigma_{r}(z)$ is given in Fig. 11. As detailed in Appendix A, by exploiting the LG expansion of eq. (4), the parameters $\sigma_{m}, \theta_{\text {rms }}$ and $z_{m}$ can be related to the coefficients $\psi_{n, \ell}$. We note that $\sigma_{m}$ may be considered as a free parameter that determines the physical transverse size of the beam. Indeed, since $w_{0}$ and $\sigma_{m}$ are related through a combination of the coefficients $\psi_{n, \ell}$, by suitably tuning $w_{0}$ it is possible to arbitrarily choose $\sigma_{m}$.

By multiplying $\sigma_{m}, \theta_{\text {rms }}$ and $k$ it is possible to obtain an adimensional quantity, the so called beam quality $\mathcal{M}_{\mathrm{rms}}^{2}$-factor [35], which is independent on the physical size of the beam. By exploiting the LG expansion of eq. (4), the $\mathcal{M}_{\mathrm{rms}}^{2}$-factor of a generic beam can be expressed as:

$$
\mathcal{M}_{\mathrm{rms}}^{2} \equiv k \theta_{\mathrm{rms}} \sigma_{m}=\sqrt{\alpha^{2}-|\beta|^{2}},
$$

where $\alpha$ and $\beta$ depend only on the expansion coefficients $\psi_{n, \ell}$ of eq. 4. Their explicit expressions are the following:

$$
\begin{aligned}
& \alpha=1+\langle|\ell|\rangle+\Phi, \\
& \beta=\sum_{n, \ell} 2 \sqrt{n(|\ell|+n)} \psi_{n, \ell} \psi_{n-1, \ell}^{*},
\end{aligned}
$$

with $\langle|\ell|\rangle=\sum_{n, \ell}|\ell|\left|\psi_{n, \ell}\right|^{2}$ and $\Phi=\sum_{n, \ell} 2 n\left|\psi_{n, \ell}\right|^{2}$. As suggested by the notation, $\langle|\ell|\rangle$ represents the mean absolute value of the OAM of the beam.

The value of $\langle|\ell|\rangle$ can be used to bound the rms divergence. As demonstrated in appendix $B$ the factor $\sqrt{\alpha^{2}-|\beta|^{2}}$ is lower bounded by $1+\langle|\ell|\rangle$. Then, the main result of our analysis can be summarized by the following bound:

$$
\mathcal{M}_{\mathrm{rms}}^{2} \geq 1+\langle|\ell|\rangle
$$

implying that the mean absolute value of the OAM increases the value of the beam divergence. We note that the well known [36, 37] inequality $\mathcal{M}_{\text {rms }}^{2} \geq 1$ for generic beams has no contribution of the orbital angular momentum as in eq. (1). Moreover, while it was well known that for a $\mathrm{LG}_{n, \ell}$ mode the beam quality factor is given by $\mathcal{M}^{2}=2 n+|\ell|+1$, it was not known what happens for a generic coherent superposition. We would like to point out that the bound was only known for incoherent superposition of LG modes 38 (with incoherent superposition we denote a beam whose intensity is given by $\left.I(x)=\sum_{n, \ell}\left|\psi_{n, \ell}\right|^{2}\left|\mathrm{LG}_{n, \ell}(x)\right|^{2}\right)$. Only in this case, the $\mathcal{M}^{2}$ factor is trivially bounded by $1+\langle|\ell|\rangle$ since for incoherent superposition we have $\mathcal{M}^{2}=\sum_{\ell} \sum_{n}(2 n+|\ell|+1)\left|\psi_{n, \ell}\right|^{2} \geq 1+\langle|\ell|\rangle$. One could ask the question whether by coherently adding different LG modes it is possible to reduce the divergence of the beam up to the standard bound $\mathcal{M}_{\text {rms }}^{2} \geq 1$. As shown by eq. (7), the answer is no, since the mean value of the OAM increases the lower bound on $\mathcal{M}^{2}$.

The bound in eq. (7) is tight, since it can be achieved by arbitrary superposition of LG modes with $n=0$. Indeed, for generic superposition $\sum_{\ell} \varphi_{\ell} \mathrm{LG}_{0, \ell}$ we have $\mathcal{M}_{\text {rms }}^{2}=1+\sum_{\ell}\left|\varphi_{\ell}\right|^{2}|\ell|=1+\langle|\ell|\rangle$, saturating the bound given by eq. (7).

This result can be easily converted to an Heisenberglike uncertainty principle (see eq. (1)). Indeed, the rms divergence $\theta_{\mathrm{rms}}$ is related to the standard deviation of the wavevectors. By defining the Fourier transform of the field as $\widetilde{\Psi}\left(k_{x}, k_{y}\right)=\frac{1}{2 \pi} \int \mathrm{d} x \mathrm{~d} y e^{i\left(k_{x} x+k_{y} y\right)} \Psi(x, y, 0)$, it is well known that, at large $z, \Psi(x, y, z) \sim$ $\frac{i k}{z} e^{i k\left(z+\frac{r^{2}}{2 z}\right)} \widetilde{\Psi}\left(\frac{k}{z} x, \frac{k}{z} y\right)$ [35]. Then, at large $z$, the radial variance can be approximated to $\sigma_{r}^{2}(z) \sim \frac{z^{2}}{k^{2}} \sigma_{k}^{2}$ with 
$\sigma_{k}^{2}=\int \mathrm{d} k_{x} \mathrm{~d} k_{y}\left(k_{x}^{2}+k_{y}^{2}\right)\left|\widetilde{\Psi}\left(k_{x}, k_{y}\right)\right|^{2}$ the variance in the Fourier space. By using the definition (2) and by noticing that $\sigma_{r} \geq \sigma_{m}$ by definition, eq. (7) can be then rewritten as

$$
\sigma_{r} \sigma_{k} \geq 1+\langle|\ell|\rangle .
$$

As already said, the bound can be applied also to microscopy. Indeed, we may exploit the well-known relationship between the Fourier transform and the images in the focal plane of thin lenses [35. In the focal plane the intensity is given by $\left|\frac{k}{f} \widetilde{\Psi}\left(\frac{k}{f} x, \frac{k}{f} y\right)\right|^{2}$. Then, the spatial extent in the focus is given by $\sigma_{\text {focus }}=\frac{f}{k} \sigma_{k}$ such that

$$
\sigma_{\text {source }} \sigma_{\text {focus }} \geq \frac{f \lambda}{2 \pi}(1+\langle|\ell|\rangle),
$$

limiting the dimension of the focused spot when the beam carries OAM. We note that the above relation should be taken into account in the coupling of OAM beam into optical fibers.

Finally, the bound (7) corresponds to an improved Heisenberg uncertainty principle. Indeed, the paraxial wave equation is mathematically equivalent to the 2DSchrödinger equation for a free particle: the direction of propagation $z$ becomes the time evolution parameter $t$ for the free particle and the wavevector $k$ is related to the particle momentum by $k=p / \hbar$. Equation (8) is then equivalent to

$$
\sigma_{r} \sigma_{p} \geq \hbar(1+\langle|\ell|\rangle),
$$

for a free-particle in 2D carrying OAM.

Eqs. (8), (9) and (10) are equivalent relations that show that the OAM becomes a fundamental quantity to study different problems, from the properties of optical beams in long-distance propagation, microscopy and optical fiber coupling to the behavior of $2 \mathrm{D}$ quantum freeparticles.

\section{A. $\mathrm{CiB}$ case}

As an example, we now explicitly evaluate the $\mathcal{M}_{\mathrm{rms}}^{2}$ factor of the Circular Beams. A generic $\mathrm{CiB}$ is determined by three complex parameters $\xi, q_{0}$ and $p$ and one integer parameter $\ell_{0} \in \mathbb{Z}$. The parameter $\xi$ is related to the beam "shape" as illustrated in Fig. 2. Specific values of $\xi$ identify some well-known beams: for instance, the limit $\xi \rightarrow+\infty$ corresponds to the LG modes, while $\mathrm{CiBs}$ with $|\xi|=1$ correspond to the generalized Hypergeometric-Gaussian modes [21, 39, 40]. The parameter $q_{0}$ is related to the physical scale (similarly to the complex beam parameter of the Gaussian beam [23]). Finally, $p$ is a radial index and $\ell_{0}$ corresponds to the carried OAM. For a circular beam, the expansion in term of LG mode was derived in 21] and it is written as

$$
\mathrm{CiB}_{p, \ell_{0}}^{\left(q_{0}, \xi\right)}(r, \phi, z)=\sum_{n=0}^{+\infty} \sum_{\ell=-\infty}^{+\infty} \psi_{n, \ell} \mathrm{LG}_{n, \ell}(r, \phi, z)
$$

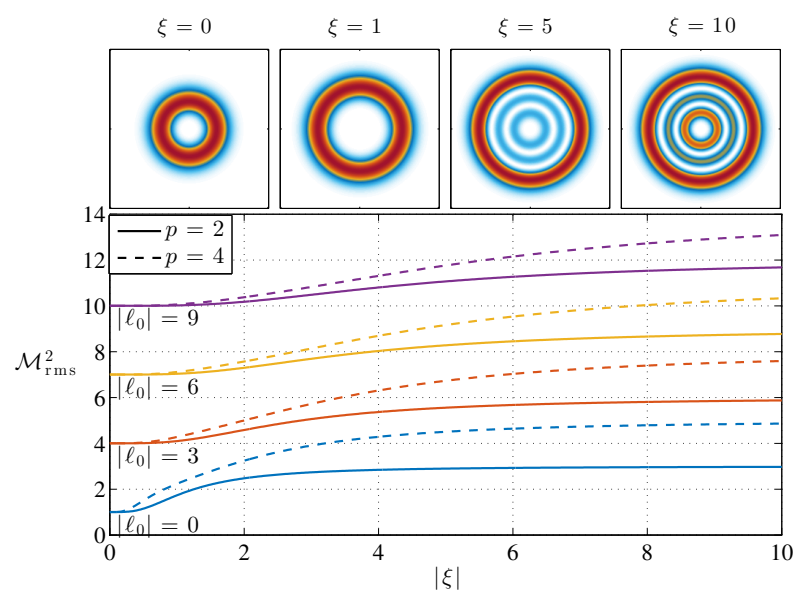

FIG. 2. (Color online) $\mathcal{M}_{\mathrm{rms}}^{2}$-factor for the CiBs with different value of $|\xi|,\left|\ell_{0}\right|$ and $p$. The lower bound $1+\left|\ell_{0}\right|$ is obtained when $\xi=0$ : in this case the $\mathrm{CiB}$ reduces to a $\mathrm{LG}_{0, \ell_{0}}$ mode. In the inset we show the intensity patterns of different $\mathrm{CiBs}$ with $\ell_{0}=3$ and $p=4$.

with

$$
\psi_{n, \ell}=\delta_{\ell, \ell_{0}} \frac{\xi^{n}}{\sqrt{\mathcal{N}}} \frac{\Gamma\left(n-\frac{p}{2}\right)}{\Gamma\left(-\frac{p}{2}\right)} \sqrt{\frac{\left|\ell_{0}\right| !}{n !\left(\left|\ell_{0}\right|+n\right) !}} .
$$

In the previous equation $\mathcal{N}$ is a normalization factor given by the Hypergeometric function $\mathcal{N}=$ ${ }_{2} F_{1}\left[-\frac{p}{2},-\frac{p^{*}}{2}, 1+\left|\ell_{0}\right|,|\xi|^{2}\right]$. For simplicity, in $\psi_{n, \ell}$ we $\operatorname{did}$ not explicitly indicate the dependence on $p, \ell_{0}$ and $\xi$.

The parameters $\Phi$ and $\beta$ that allow to calculate the beam quality factor can be explicitly evaluated from 12 and their value is given by $\Phi=\frac{1}{\mathcal{N}} \frac{|p \xi|^{2}}{2+2\left|\ell_{0}\right|}{ }_{2} F_{1}\left[1-\frac{p}{2}, 1-\right.$ $\left.\frac{p^{*}}{2}, 2+\left|\ell_{0}\right|,|\xi|^{2}\right]$ and $\beta=\xi(\Phi-p)$. The mean absolute value of the OAM is simply $\langle|\ell|\rangle=\left|\ell_{0}\right|$. Then, the $\mathcal{M}_{\mathrm{rms}^{-}}^{2}$ factor depends only on $|\xi|, p$ and $\left|\ell_{0}\right|$ as

$$
\mathcal{M}_{\mathrm{rms}}^{2}=\sqrt{\left(1+\left|\ell_{0}\right|+\Phi\right)^{2}-|\xi(\Phi-p)|^{2}} .
$$

The behavior of $\mathcal{M}_{\mathrm{rms}}^{2}$ is shown in Fig. 2 for different values of $\ell_{0}$ and $p=2$ or $p=4$. In particular, when $|\xi|=1$, the $\mathcal{M}_{\text {rms }}^{2}$-factor has a simple expression:

$$
\mathcal{M}_{\mathrm{rms}}^{2}=\sqrt{\left(1+\left|\ell_{0}\right|\right)^{2}+\frac{|p|^{2}}{\Re \mathrm{e}(p)+\left|\ell_{0}\right|}} .
$$

Since the rms divergence can be defined in this case only when $\Re \mathrm{e}(p)>-\left|\ell_{0}\right|$ (see [21), it can be easily checked that quality factor expressed in eq. 14 satisfies the general bound of eq. (7).

\section{ENCIRCLED-ENERGY DIVERGENCE}

As already noted in 21, the CiBs with $|\xi|=1$ and $-\left|\ell_{0}\right|-1<\Re \mathrm{e}(p) \leq-\left|\ell_{0}\right|$ are square integrable beams 


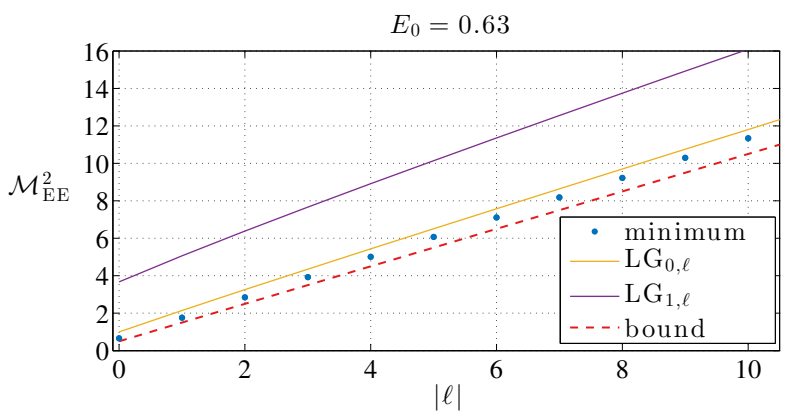

FIG. 3. (Color online) Minimum values of $\mathcal{M}_{\mathrm{EE}}^{2} \equiv k \theta_{\mathrm{EE}} R_{m}$ calculated over random beams with fixed value of $\ell$. We used $E_{0} \simeq 0.63$. We compare the minimum values of $\mathcal{M}_{\mathrm{EE}}^{2}$ with the ones obtained with the $\mathrm{LG}_{0, \ell}$ and $\mathrm{LG}_{1, \ell}$ modes. Dashed line represents the lower bound as given in eq. (18).

but their rms divergence cannot be defined. This feature is common to all fields whose intensity (at large $r$ and fixed $z$ ) fall-off as $1 / r^{2+a}$, with $0<a \leq 2$. For this reason, in these cases an alternative expression of the divergence should be used. To this purpose, we may define the so-called Encircled-Energy far field angle of divergence $\theta_{\mathrm{EE}}$ by

$$
\theta_{\mathrm{EE}}=\lim _{z \rightarrow+\infty} \frac{R_{\mathrm{EE}}(z)}{z},
$$

where $R_{\mathrm{EE}}(z)$ is the Encircled-Energy radius. $R_{\mathrm{EE}}(z)$ must be calculated by the implicit relation $\int \mathrm{d} \phi \int_{0}^{R_{\mathrm{EE}}(z)} \mathrm{d} r r I(r, \phi, z)=E_{0}$, with $E_{0}$ a fixed constant. Here, $R_{\mathrm{EE}}(z)$ corresponds to the radius whose corresponding circle centered on the beam axis contains a given fraction $E_{0}$ of the total beam energy. By definition, the divergence $\theta_{\mathrm{EE}}$ is well defined for any square integrable beam.

We now show that a bound similar to 77 can be obtained for $\theta_{\mathrm{EE}}$, with $\sigma_{m}$ replaced by $R_{m} \equiv \min _{z} R(z)$, the minimum of the encircled-energy radius. As it happens for the rms divergence, the product $\mathcal{M}_{\mathrm{EE}}^{2} \equiv k \theta_{\mathrm{EE}} R_{m}$ is adimensional and depends only on the expansion coefficients $\psi_{n, \ell}$. As detailed in appendix B, it is possible to show that

$$
\mathcal{M}_{\mathrm{EE}}^{2}=\sqrt{T_{\infty} \min _{Z}\left[\left(1+Z^{2}\right) T(Z)\right]},
$$

with $T_{\infty} \equiv \lim _{Z \rightarrow \infty} T(Z)$. The function $T(Z)$ is defined only in terms of the expansion coefficient by the implicit relation

$$
E_{0}=\sum_{\ell} \int_{0}^{T(Z)}\left|\mathcal{U}_{\ell}(t, Z)\right|^{2} \mathrm{~d} t,
$$

where $\mathcal{U}_{\ell}(t, Z)=\sum_{n=0}^{\infty} \sqrt{\frac{n !|t| \ell \mid}{(|\ell|+n) !}} \psi_{n, \ell}\left(\frac{1+i Z}{1-i Z}\right)^{n} e^{-\frac{t}{2}} L_{n}^{(|\ell|)}(t)$.

We numerically evaluated the minimum of $\mathcal{M}_{\mathrm{EE}}^{2}$ for a fixed value of OAM $\ell$. For each value of $\ell$, we searched for the minimum of $\mathcal{M}_{\mathrm{EE}}^{2}$ by using a truncated superposition (up to $n=10$ ) of the $\mathrm{LG}_{n, \ell}$ modes as in eq.

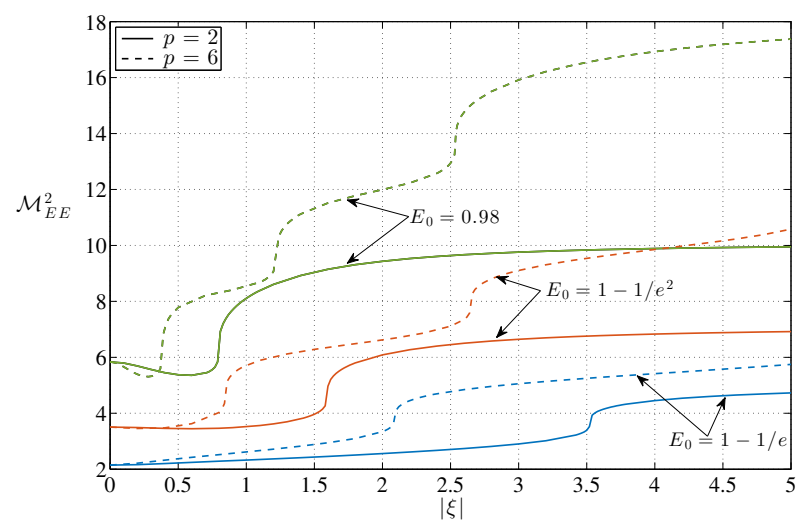

FIG. 4. (Color online) Encircled-Energy divergence of CiBs for different values of $E_{0}$. We plot the product $\mathcal{M}_{\mathrm{EE}}^{2} \equiv$ $k \theta_{\mathrm{EE}} R_{m}$ in function of $|\xi|$ (for real $\xi$ ) with $\ell=1$ and $p=2$ or $p=6$. By increasing $E_{0}$, the value of $\mathcal{M}_{\mathrm{EE}}^{2}$ increases.

(4). We fixed $E_{0}=1-1 / e \simeq 0.63$ : such value is required to achieve $\theta_{\mathrm{EE}}=\theta_{\mathrm{rms}}$ for the Gaussian beams (i.e. the $\mathrm{LG}_{0,0}$ mode). Different values of $E_{0}$ will be discussed later. The results of the numerical minimization, performed by using the Nelder-Mead algorithm 44, are shown in Fig. 3 for different values of $\ell$. For comparison, we also show the value of $k \theta_{\mathrm{EE}} R_{m}$ for the $\mathrm{LG}_{0, \ell}$ and $\mathrm{LG}_{1, \ell}$ modes. The minimum of $k \theta_{\mathrm{EE}} R_{m}$ for a random beam is slightly lower than the value obtained for the $\mathrm{LG}_{0, \ell}$ mode, but it cannot be arbitrarily low. Indeed, our numerical minimization shows that the divergence $\theta_{\mathrm{EE}}$ satisfies the following bound for any beam with a fixed value of OAM given by $\ell$ :

$$
\theta_{\mathrm{EE}} \geq \frac{1}{k R_{m}}\left(c_{0}+|\ell|\right),
$$

with $c_{0}=0.5$. In figure Fig. 3 we report such bound by using a dashed line.

The value $E_{0} \simeq 0.63$ may seem arbitrary and indeed the value of the product $k \theta_{\mathrm{EE}} R_{m}$ depends on such choice. For instance, as illustrated in Fig. 4 for the CiBs, by increasing the value of $E_{0}$, the value of $\mathcal{M}_{\mathrm{EE}}^{2}$ will increase. In order to check the validity of the bound in eq. (18), we tested it for different values of $E_{0}$, obtaining the same bound (18) with different values of $c_{0}$ : in particular, for $E_{0}=1-1 / e^{2} \simeq 0.86$ (a common value used to define the divergence) we obtained $c_{0}=1.8$ while for $E_{0}=$ 0.98 we obtained $c_{0}=3$. Details of the minimization procedure and the results for $E_{0}=0.86$ and $E_{0}=0.98$ are presented in appendix C. In appendix D we also show some examples of beams that minimize $\mathcal{M}_{\mathrm{EE}}^{2}$.

We conjecture that, as long as $E_{0}>0.5$, the bound (18) holds. The parameter $c_{0}$ will depends on the specific value chosen for $E_{0}$. The conjecture is based on the fact that, at large $|\ell|$, the asymptotic divergence of the $\mathrm{LG}_{0, \ell}$ modes is given by $\mathcal{M}_{\mathrm{EE}}^{2} \sim|\ell|+t \sqrt{2|\ell|}$ with $t=\operatorname{erf}^{-1}\left(2 E_{0}-1\right)$ (see details in appendix C). When $E_{0}>0.5$ we have $t>0$ and the $\mathcal{M}^{2}$ parameter for the $\mathrm{LG}_{0, \ell}$ is always larger than $|\ell|$. 


\section{CONCLUSIONS}

We studied the divergence of generic beams carrying OAM. We demonstrated that the rms divergence is bounded by the absolute mean value of the beam orbital angular momentum (see eq. (7)). We have shown that such bound provides an uncertainty relation that is useful for applications involving long-distance propagation of beams and in the study of vorticity generation, in the limits of imaging and illumination in microscopy as well as in the study of quantum free-particle confined in 2D (see eq. (8), (9) and (10)). Finally, we demonstrated that a similar bound holds for a different definition of the divergence, the so called Encircled-Energy divergence. Our results prove that the presence of orbital angular momentum enhances the angular spreading of the beam, leading to an increase of the uncertainty relation between the spatial and wavevector extent. Such uncertainty implies an increased beam divergence and a lower ability in focusing the beam.

Our results are obtained for paraxial beam. A recent work 42. showed that a similar bound, $k R_{\mathrm{rms}} \sin \theta_{\mathrm{rms}} \geq$ $\cos \theta_{\mathrm{rms}}+\left|\left\langle L_{z}\right\rangle\right|$, can be achieved for non-paraxial electromagnetic beams. However, the bound was shown only for a combination of two eigenstates of the OAM operator $L_{z}=-i \frac{\partial}{\partial \phi}$. We note that the result obtained in 42 ] reduce to eq. (7) for OAM eigenstates and small $\theta$ (i.e. paraxial beam). Our technique, combined to the results obtained in 42] may allow to investigate the divergence properties of generic non-paraxial beams carrying OAM. We leave such investigation for future works.

\section{ACKNOWLEDGMENTS}

G.V. and P.V. acknowledge the Strategic-ResearchProject QUINTET of the Department of Information Engineering, University of Padova, and the Progetto di Ateneo PRAT 2013 "OAM in free space: a new resource for QKD" (CPDA138592). G.P., E.M., F.S. and F.T. acknowledge the support and collaboration of SIAE Microelectronics.

\section{Appendix A: Evaluation of $\sigma_{r}(z)$}

In this section we analytically evaluate the beam variance $\sigma_{r}^{2}(z)$ and the rms divergence $\theta_{\text {rms }}$ of a generic beam. We consider a generic paraxial beam $\Psi(r, \phi, z)$ propagating in the $z$ direction. The beam can be expanded in the basis of the LG modes as follows:

$$
\Psi(r, \phi, z)=\sum_{n=0}^{+\infty} \sum_{\ell=-\infty}^{+\infty} \psi_{n, \ell} \mathrm{LG}_{n, \ell}(r, \phi, z),
$$

with $\sum_{n=0}^{+\infty} \sum_{\ell=-\infty}^{+\infty}\left|\psi_{n, \ell}\right|^{2}=1$.
Our convention for the (normalized) LG modes with beam waist parameter $w_{0}$ is the following:

$$
\begin{aligned}
\mathrm{LG}_{n, \ell}= & \sqrt{\frac{2}{\pi}} \sqrt{\frac{n !}{(|\ell|+n) !}} \frac{e^{-\frac{i k r^{2}}{2 q(z)}}}{w(z)}\left(\frac{\sqrt{2} r}{w(z)}\right)^{|\ell|} \times \\
& L_{n}^{(|\ell|)}\left(\frac{2 r^{2}}{w^{2}(z)}\right) e^{i \ell \phi} e^{i(2 n+|\ell|+1) \zeta(z)},
\end{aligned}
$$

where $n, \ell \in \mathbb{Z}$ with $n \geq 0, L_{n}^{(|\ell|)}(x)$ is the generalized Laguerre polynomial, $w(z)=w_{0} \sqrt{1+\left(z / z_{0}\right)^{2}}$ is the beam size, $z_{0}=k w_{0}^{2} / 2$ is the Rayleigh range and $\exp [i \zeta(z)]=\left(z_{0}+i z\right) /\left|z_{0}+i z\right|$ is the Gouy phase. The integers $n$ and $\ell$ respectively represent the radial quantum number and the OAM content of each LG mode. We note that the physical scale of the beam $\Psi$ in eq. A1 is determined by the value of the beam waist parameter $w_{0}$.

The rms variance $\sigma_{r}^{2}(z)$ of the beam $\Psi$ is defined in eq. (3) of the main text. By using the LG mode expansion, the variance may be rewritten as:

$$
\begin{aligned}
\sigma_{r}^{2}(z) & =\int_{0}^{2 \pi} \mathrm{d} \phi \int_{0}^{+\infty} \mathrm{d} r r^{3}|\Psi(r, \phi, z)|^{2} \\
& =\sum_{n, n^{\prime}, \ell, \ell^{\prime}} \psi_{n, \ell} \psi_{n^{\prime}, \ell^{\prime}}^{*} \int_{0}^{2 \pi} \mathrm{d} \phi \int_{0}^{+\infty} \mathrm{d} r r^{3} \mathrm{LG}_{n, \ell} \mathrm{LG}_{n^{\prime}, \ell^{\prime}}^{*}
\end{aligned}
$$

The above integral on the LG modes is evaluated as [21]:

$$
\begin{aligned}
& \int_{0}^{2 \pi} \mathrm{d} \phi \int_{0}^{+\infty} \mathrm{d} r r^{3} \mathrm{LG}_{n, \ell}(r, \phi, z) \mathrm{LG}_{n^{\prime}, \ell^{\prime}}^{*}(r, \phi, z)= \\
& \frac{\delta_{\ell, \ell^{\prime}}}{2}\left[B_{\ell, n}(z) \delta_{n^{\prime}, n}-C_{\ell, n}(z) \delta_{n^{\prime}+1, n}-C_{\ell, n^{\prime}}^{*}(z) \delta_{n^{\prime}, n+1}\right]
\end{aligned}
$$

with $B_{\ell, n}=w^{2}(z)(|\ell|+2 n+1)$ and $C_{\ell, n}=$ $w^{2}(z) e^{2 i \zeta(z)} \sqrt{n(|\ell|+n)}$.

By plugging the above result into eq. A3 it is possible to obtain an explicit expression for $\sigma_{\mathrm{rms}}^{2}(z)$, namely:

$$
\sigma_{\mathrm{rms}}^{2}(z)=\frac{w^{2}(z)}{2}\left[1+\langle|\ell|\rangle+\Phi-\Re \mathrm{e}\left(e^{2 i \zeta(z)} \beta\right)\right],
$$

where $\langle|\ell|\rangle, \Phi$ and $\beta$ are parameters that depend on the expansion coefficient $\psi_{n, \ell}$ as follows:

$$
\begin{aligned}
\langle|\ell|\rangle & =\sum_{\ell=-\infty}^{+\infty} \sum_{n=0}^{\infty}|\ell|\left|\psi_{n, \ell}\right|^{2}, \\
\Phi & \equiv \sum_{\ell=-\infty}^{+\infty} \sum_{n=0}^{\infty} 2 n\left|\psi_{n, \ell}\right|^{2}, \\
\beta & =\sum_{\ell=-\infty}^{+\infty} \sum_{n=0}^{\infty} 2 \sqrt{n(|\ell|+n)} \psi_{n, \ell} \psi_{n-1, \ell}^{*} .
\end{aligned}
$$

Since $w(z)=w_{0} \sqrt{1+\left(z / z_{0}\right)^{2}}$ and $\exp [i \zeta(z)]=\left(z_{0}+\right.$ $i z) /\left|z_{0}+i z\right|$, by defining $\alpha=1+\langle|\ell|\rangle+\Phi$, it is possible to 
show that the variance $\sigma_{r}^{2}(z)$ has a parabolic dependence on $z$, namely:

$$
\sigma^{2}(z)=\sigma_{m}^{2}+\theta_{\mathrm{rms}}^{2}\left(z-z_{m}\right)^{2},
$$

with

$$
\begin{aligned}
z_{m} & =-z_{0} \frac{\Im \mathrm{m} \beta}{\Re \mathrm{e}(\alpha+\beta)}, \\
\sigma_{m}^{2} & =\frac{w_{0}^{2}}{2} \frac{\alpha^{2}-|\beta|^{2}}{\Re \mathrm{e}(\alpha+\beta)}, \\
\theta_{\mathrm{rms}} & =\frac{w_{0}}{\sqrt{2} z_{0}} \sqrt{\Re \mathrm{e}(\alpha+\beta)} .
\end{aligned}
$$

Since the transverse scale can be fixed by $w_{0}$ or equivalently by $\sigma_{m}$, we can express $\theta_{\text {rms }}$ and $z_{m}$ in function of $\sigma_{m}$, obtaining

$$
\begin{aligned}
\theta_{\mathrm{rms}} & =\frac{1}{k \sigma_{m}} \sqrt{\alpha^{2}-|\beta|^{2}}, \\
z_{m} & =-k \sigma_{m}^{2} \frac{\Im \mathrm{m} \beta}{\alpha^{2}-|\beta|^{2}}=-\frac{\Im \mathrm{m} \beta}{k \theta_{\mathrm{rms}}^{2}} .
\end{aligned}
$$

We have thus demonstrated eqs. (5) and (6) of the main text.

\section{Appendix B: Proof of the bound}

In this section we demonstrate that the following bound holds:

$$
\mathcal{M}_{\mathrm{rms}}^{2} \equiv k \theta_{\mathrm{rms}} \sigma_{m} \geq 1+\langle|\ell|\rangle .
$$

In the previous section we have shown that, for a generic beam, $k \theta_{\text {rms }} \sigma_{m}=\sqrt{\alpha^{2}-|\beta|^{2}}$, with $\alpha=\sum_{\ell} \sum_{n}(1+2 n+$ $|\ell|)\left|\psi_{n, \ell}\right|^{2}$ and $\beta=\sum_{\ell} \sum_{n} 2 \sqrt{n(|\ell|+n)} \psi_{n, \ell} \psi_{n-1, \ell}^{*}$. Our goal is to find a lower bound for $\sqrt{\alpha^{2}-|\beta|^{2}}$ depending on the average value of the OAM.

If we define $\widetilde{\beta}=\sum_{\ell} \sum_{n} 2 \sqrt{n(|\ell|+n)}\left|\psi_{n, \ell} \psi_{n-1, \ell}\right|$, by the properties of the absolute value, it follows that $|\beta| \leq$ $\widetilde{\beta}$ and:

$$
\alpha^{2}-|\beta|^{2} \geq \alpha^{2}-\widetilde{\beta}^{2} .
$$

We now define $\Delta^{ \pm}=\sum_{\ell} \sum_{n=0}^{\infty}\left(\sqrt{|\ell|+n+1}\left|\psi_{n, \ell}\right| \pm\right.$ $\left.\sqrt{n+1}\left|\psi_{n+1, \ell}\right|\right)^{2}$. By expanding the square in $\Delta^{ \pm}$it possible to show that

$$
\Delta^{ \pm}=\alpha \pm \widetilde{\beta}
$$

Then

$$
\alpha^{2}-\widetilde{\beta}^{2}=(\alpha+\widetilde{\beta})(\alpha-\widetilde{\beta})=\Delta^{+} \Delta^{-} .
$$

We note that $\Delta^{ \pm}$can be written as the expectation value of operators in a real valued vector space. Let's consider an infinite dimensional space spanned by the orthonormal vectors $\{|n, \ell\rangle\}$ with $n=0, \cdots,+\infty$ and

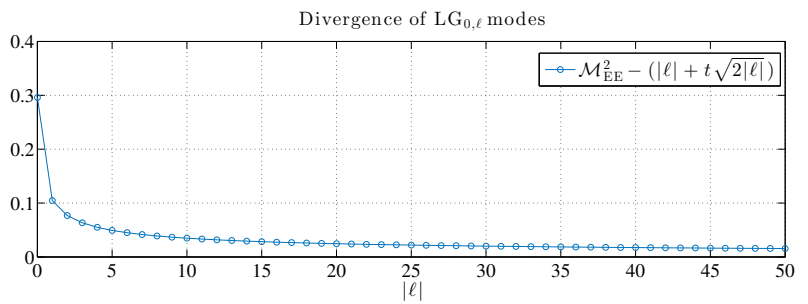

FIG. 5. (Color online) Difference between the $\mathcal{M}_{\mathrm{EE}}^{2}$ value and its approximaton, $|\ell|+t \sqrt{2|\ell|}$, for the $\mathrm{LG}_{0, \ell}$ modes.

$\ell=-\infty, \cdots,+\infty$ and define the following operators $\hat{N}$, $\hat{L}, \hat{a}$ and $\hat{a}^{\dagger}$ by their action on the basis states:

$$
\begin{aligned}
& \hat{N}|n, \ell\rangle=n|n, \ell\rangle, \quad \hat{a}|n, \ell\rangle=\sqrt{n}|n-1, \ell\rangle, \\
& \hat{L}|n, \ell\rangle=\ell|n, \ell\rangle, \quad \hat{a}^{\dagger}|n, \ell\rangle=\sqrt{n+1}|n+1, \ell\rangle \text {. }
\end{aligned}
$$

If we define $\hat{A}_{ \pm}=\sqrt{\hat{N}+|\hat{L}|+1} \pm \hat{a}$ and a generic vector $|v\rangle$ as $|v\rangle=\sum_{l} \sum_{n}\left|\psi_{n, \ell}\right||n, \ell\rangle$, we have

$$
\hat{A}_{ \pm}|v\rangle=\sum_{n, \ell}\left[\sqrt{n+|\ell|+1}\left|\psi_{n, \ell}\right| \pm \sqrt{n+1}\left|\psi_{n+1, \ell}\right|\right]|n, \ell\rangle
$$

Since the vectors $|n, \ell\rangle$ are orthonormal and thus satisfy $\left\langle n^{\prime}, \ell^{\prime} \mid n, \ell\right\rangle=\delta_{n, n^{\prime}} \delta_{\ell, \ell^{\prime}}$ the expectation value of $A_{ \pm}^{\dagger} A_{ \pm}$ are precisely $\Delta^{ \pm}$:

$$
\left\langle v\left|A_{ \pm}^{\dagger} A_{ \pm}\right| v\right\rangle=\Delta^{ \pm} .
$$

By the triangular inequality we may then bound $\Delta^{+} \Delta^{-}$:

$$
\Delta^{+} \Delta^{-}=\left\langle v\left|A_{+}^{\dagger} A_{+}\right| v\right\rangle\left\langle v\left|A_{-}^{\dagger} A_{-}\right| v\right\rangle \geq\left|\left\langle v\left|A_{+}^{\dagger} A_{-}\right| v\right\rangle\right|^{2}
$$

The r.h.s of the previous equation can be explicitely evaluated to give:

$$
\left|\left\langle v\left|A_{+}^{\dagger} A_{-}\right| v\right\rangle\right|^{2}=(1+\langle|\ell|\rangle)^{2} .
$$

By combining eqs. (B2), (B4), (B8) and (B9), it follows that

$$
k \theta_{\mathrm{rms}} \sigma_{m} \geq 1+\langle|\ell|\rangle
$$

\section{Appendix C: Encircled-energy divergence}

Here we evaluate the encircled-energy divergence in function of the expansion coefficient $\psi_{n, \ell}$. We consider a generic paraxial beam expanded as a superposition of LG modes, as in equation (A1). The integral $\int \mathrm{d} \phi \int_{0}^{R_{\mathrm{EE}}(z)} \mathrm{d} r r I(x, y, x)=E_{0}$ defining the encircledenergy radius $R(z)$ can be written in adimensional notation as

$$
E_{0}=\int_{0}^{T(Z)} \sum_{\ell}\left|\mathcal{U}_{\ell}(t, Z)\right|^{2} \mathrm{~d} t
$$



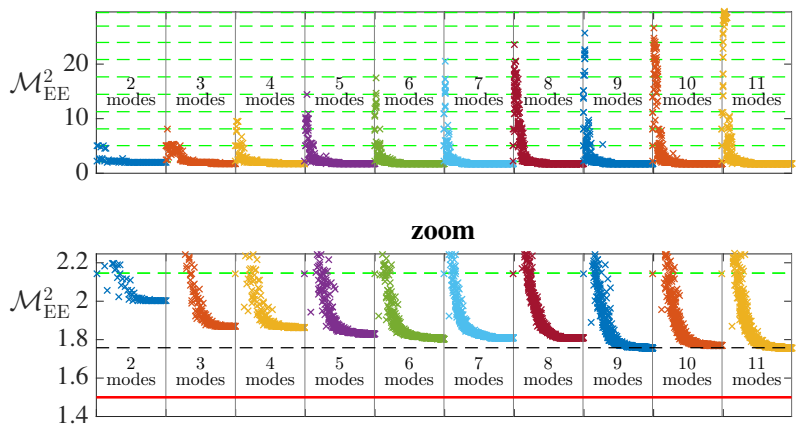

FIG. 6. (Color online) Steps of the Nelder-Mead used to minimize $\mathcal{M}_{\mathrm{EE}}^{2}$. We used $\ell=1$ and $E_{0} \simeq 0.63$. We report the number of LG modes used in the superposition of eq. (D1). With green (light gray) dashed lines we show the $\mathcal{M}_{\mathrm{EE}}^{2}$ values of the $\mathrm{LG}_{n, 1}$ modes, while the black dashed line is the minimum $\mathcal{M}_{\mathrm{EE}}^{2}$ achieved. The continuous red (dark gray) line represents the bound $c_{0}+|\ell|$ with $c_{0}=0.5$.

with $Z=z / z_{0}$ and

$$
\begin{aligned}
\mathcal{U}_{\ell}(t, Z) & =\sum_{n=0}^{\infty} \sqrt{\frac{e^{-t} t^{|\ell|} n !}{(|\ell|+n) !}} \psi_{n, \ell}\left(\frac{1+i Z}{1-i Z}\right)^{n} L_{n}^{(|\ell|)}(t) \\
T(Z) & =\frac{2 R_{\mathrm{EE}}^{2}\left(Z z_{0}\right)}{w_{0}^{2}\left(1+Z^{2}\right)}
\end{aligned}
$$

The minimum $R_{m}$ of $R_{E E}(z)$ should be found by minimizing the function $\sqrt{\left(1+Z^{2}\right) T_{\mathrm{EE}}(Z)}$. We define $T_{\infty} \equiv \lim _{Z \rightarrow \infty} T(Z)$, namely

$$
E_{0}=\int_{0}^{T_{\infty}} \sum_{\ell}\left|\mathcal{U}_{\ell}^{\infty}(t)\right|^{2} \mathrm{~d} t
$$

and $\mathcal{U}_{\ell}^{\infty}(t)=\sqrt{e^{-t} t^{|m|}} \sum_{n=0}^{N} \sqrt{\frac{n !}{(|m|+n) !}} \psi_{n}(-1)^{n} L_{n}^{(m)}(t)$. By using the definition of $T(Z)$ in eq. C2 it is possible to show that $T_{\infty}=\frac{2 z_{0}^{2}}{w_{0}^{2}} \theta_{\mathrm{EE}}^{2}$. By using $z_{0}=k w_{0}^{2} / 2$ and $R_{m} / w_{0}=\sqrt{\min _{Z}\left[\left(1+Z^{2}\right) T(Z)\right] / 2}$ we may express $\theta_{\mathrm{EE}}$ as

$$
\begin{aligned}
\theta_{\mathrm{EE}} & =\frac{\sqrt{2}}{k w_{0}} \sqrt{T_{\infty}} \\
& =\frac{1}{k R_{m}} \sqrt{T_{\infty} \min _{Z}\left[\left(1+Z^{2}\right) T(Z)\right]} .
\end{aligned}
$$

Also in this case the product $\mathcal{M}_{\mathrm{EE}}^{2} \equiv k \theta_{\mathrm{EE}} R_{m}$ is adimensional and depends only on the expansion coefficient $\psi_{n, \ell}$

The above relations can be simplified for LG modes. Indeed, for an LG mode, $T(Z)$ is an even function of $Z$ and its minimum is obtained at $Z=0$. Moreover we have $T(Z)=T(0)=T_{\infty} \equiv T_{n, \ell}$. Then the divergence for a $L G_{n, \ell}$ mode can be expressed as

$$
\theta_{\mathrm{EE} n, \ell}=\frac{1}{k R_{m}} T_{n, \ell},
$$
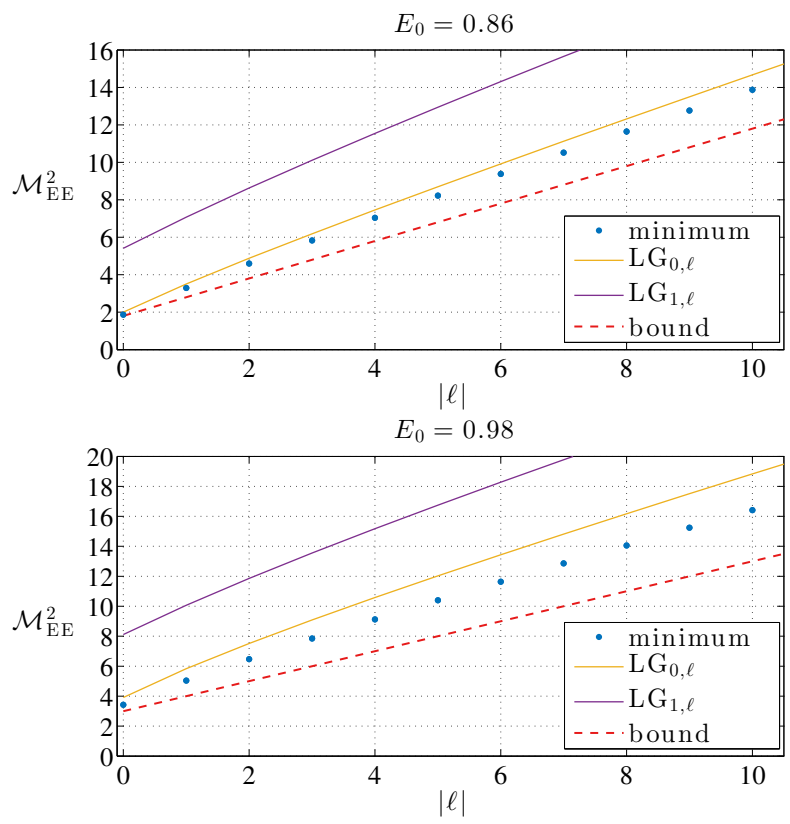

FIG. 7. (Color online) Minimum values of $\mathcal{M}_{\mathrm{EE}}^{2}$ calculated over beams with fixed value of $\ell$. We used $E_{0} \simeq 0.86$ in the upper graph and $E_{0}=0.98$ in the lower graph.

with

$$
E_{0}=\frac{n !}{(|\ell|+n) !} \int_{0}^{T_{n, \ell}} e^{-t} t^{|\ell|}\left(L_{n}^{(|\ell|)}(t)\right)^{2} \mathrm{~d} t .
$$

The divergence of the $\mathrm{LG}_{0, \ell}$ modes can be further simplified and it is expressed through special functions: in this case, equation (C6) can written as $P\left(|\ell|+1, T_{0, \ell}\right)=$ $E_{0}$, where $P(a, z)$ is the regularized incomplee Gamma function $P(a, z)=\frac{1}{\Gamma(a)} \int_{0}^{z} t^{a-1} e^{-t} \mathrm{~d} t$. By using a formula due to Tricomi [43] the asymptotic behavior at large $|\ell|$ of $P(a, z)$ is the following:

$$
P(|\ell|+1,|\ell|+t \sqrt{2|\ell|})=\frac{1}{2}[|1|+\operatorname{erf}(t)]+O\left(\frac{1}{\sqrt{|\ell|}}\right),
$$

with $\operatorname{erf}(t)$ the error function. Then, at large $|\ell|$, the asymptotic divergence of the $\mathrm{LG}_{0, \ell}$ mode is

$$
\theta_{0, \ell}^{(E E)} \sim \frac{1}{k R_{m}}(|\ell|+t \sqrt{2|\ell|}),
$$

with $t=\operatorname{erf}^{-1}\left(2 E_{0}-1\right)$. Note that when $E_{0}=\frac{1}{2}+\epsilon$ we have $t \sim \sqrt{\pi} \epsilon$. The r.h.s. of eq. (C8) is a good approximation of the divergence also for low values of $|\ell|$, as demonstrated by Fig. 5 .

\section{Appendix D: Numerical minimization}

As described in the main text, we numerically evaluated the minimum of $\mathcal{M}_{\mathrm{EE}}^{2}$ at fixed value of OAM $\ell$. Here we illustrated with more details the method. For 

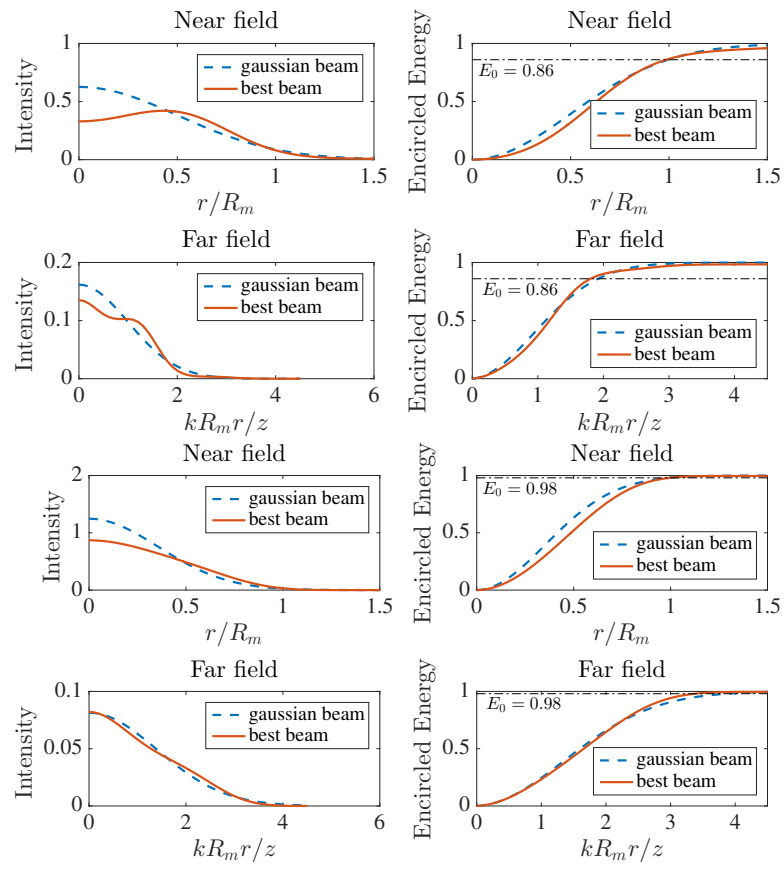

FIG. 8. (Color online) Intensity patterns and encircled-energy for the Gaussian beam and the best beam at $\ell=0$ that minimize $\mathcal{M}_{\mathrm{EE}}^{2}$. The physical size of the beams are chosen such that they have the same value of $R_{m}$. With dash-dot lines we indicate the value of $E_{0}$ that define the encircledenergy radius $R(z)$. The optimal beam is obtained for $E_{0}=$ 0.86 for the two upper rows figures, while $E_{0}=0.98$ in the two bottom rows. each value of $\ell$, we defined the generic wavefunction as a truncated superposition of the first $N \mathrm{LG}_{n, \ell}$ modes:

$$
\Psi_{\ell}\left(\left\{\psi_{n}\right\}\right)=\sum_{n=0}^{N-1} \psi_{n} \mathrm{LG}_{n, \ell}(r, \phi, z) .
$$

The wavefunction is uniquely determined by the coefficients $\left\{\psi_{0}, \psi_{1}, \cdots, \psi_{N-1}\right\}$. Due to the equivalence under a global phase, we set $\psi_{0} \in \mathbb{R}$, while we considered the remaining coefficients as complex. By using the NelderMead algorithm [44, we search for the coefficients $\left\{\psi_{n}\right\}$ that minimize the $\mathcal{M}_{\mathrm{EE}}^{2}$. To take into account the normalization $\sum_{n}\left|\psi_{n}\right|^{2}=1$, we adapted the algorithm to an hypersphere. We here recall that the $\mathcal{M}_{\mathrm{EE}}^{2}$ parameter is defined in eq. (13) of the main text. In fig. 6 we show the iterations of the algorithm for different truncation $N$ and for $\ell=1$ and $E_{0} \simeq 0.63$. By increasing the number of modes, the minimum value of $\mathcal{M}_{\mathrm{EE}}^{2}$ decreases but it is always larger than the bound $c_{0}+|\ell|$ with $c_{0}=0.5$. The procedure was repeated for different values of $\ell$ to obtain the graph shown in fig. 3 in the main text.

We also performed the minimization for different values of $E_{0}$. The results are presented in Fig. 7 for $E_{0} \simeq 0.86$ and $E_{0}=0.98$. In both cases the bound $c_{0}+|\ell|$ holds with $c_{0}=1.8$ and $c_{0}=3$ respectively.

Finally, in Fig. 8, for $\ell=0$ we show the intensity patterns of the best divergence beam and the comparison with the gaussian beam (namely the lowest LG mode) and $E_{0}=0.86$ and $E_{0}=0.98$.
[1] L. Allen, M. W. Beijersbergen, R. J. C. Spreeuw, and J. P. Woerdman, Phys. Rev. A 45, 8185 (1992).

[2] S. Fürhapter, A. Jesacher, S. Bernet, and M. RitschMarte, Opt. Exp. 13, 689 (2005).

[3] D. G. Grier, Nature 424, 810 (2003).

[4] G. Anzolin, F. Tamburini, A. Bianchini, G. Umbriaco, and C. Barbieri, Astronomy and Astrophysics 488, 1159 (2008).

[5] E. Mari, F. Tamburini, G. a. Swartzlander, A. Bianchini, C. Barbieri, F. Romanato, and B. Thidé, Optics Express 20, 2445 (2012).

[6] F. Tamburini, E. Mari, A. Sponselli, B. Thidé, A. Bianchini, and F. Romanato, New Journal of Physics 14, 033001 (2012).

[7] J. Wang, et al., Nat. Phot. 6, 488 (2012).

[8] N. Bozinovic, Y. Yue, Y. Ren, M. Tur, P. Kristensen, H. Huang, A. E. Willner, and S. Ramachandran, Science (New York, N.Y.) 340, 1545 (2013).

[9] G. Vallone, V. D'Ambrosio, A. Sponselli, S. Slussarenko, L. Marrucci, F. Sciarrino, and P. Villoresi, Phys. Rev. Lett. 113, 060503 (2014).

[10] M. M. Mohammad Mirhosseini, Omar S Magaña-Loaiza, Malcolm N O'Sullivan, Brandon Rodenburg, M. P. J. Lavery, M. J. Padgett, D. J. Gauthier, and R. W. Boyd, New Journal of Physics 17, 033033 (2015).

[11] M. Merano, A. Aiello, M. P. van Exter, and J. P. Woerdman, Nature Photonics 3, 337 (2009).
[12] F. Tamburini, B. Thidé, G. Molina-Terriza, and G. Anzolin, Nat. Phys. 7, 195 (2011).

[13] V. D'Ambrosio, F. Bisesto, F. Sciarrino, J. F. Barra, G. Lima, and A. Cabello, Physical Review Letters 112, 140503 (2014).

[14] Y. Yan, et al., Nature Communications 5, 4876 (2014).

[15] F. Tamburini, E. Mari, G. Parisi, F. Spinello, M. Oldoni, R. A. Ravanelli, P. Coassini, C. G. Someda, B. Thidé, and F. Romanato, Radio Science 50, 501 (2015).

[16] R. L. Phillips and L. C. Andrews, Applied Optics 22, 643 (1983).

[17] A. E. Siegman, IEEE Journal of Quantum Electronics 27, 1146 (1991).

[18] G. Parisi, E. Mari, F. Spinello, F. Romanato, and F. Tamburini, Optics Express 22, 17135 (2014).

[19] M. J. Padgett, F. M. Miatto, M. P. J. Lavery, A. Zeilinger, and R. W. Boyd, New Journal of Physics 17, 023011 (2015).

[20] S. G. Reddy, C. Permangatt, S. Prabhakar, A. Anwar, J. Banerji, and R. P. Singh, Applied Optics 54, 6690 (2015).

[21] G. Vallone, Opt. Lett. 40, 1717 (2015).

[22] M. A. Bandres and J. C. Gutiérrez-Vega, Optics Letters 33, 177 (2008).

[23] A. E. Siegman, Lasers, University Science (1986).

[24] A. Wünsche, Journal of the Optical Society of America A 6, 1320 (1989). 
[25] J. C. Gutiérrez-Vega and M. A. Bandres, Journal of the Optical Society of America A 22, 289 (2005).

[26] M. V. Berry, Journal of Optics A: Pure and Applied Optics 6, 259 (2004).

[27] L. Marrucci, C. Manzo, and D. Paparo, Phys. Rev. Lett. 96, 163905 (2006).

[28] M. Beijersbergen, R. Coerwinkel, M. Kristensen, and J. Woerdman, Optics Communications 112, 321 (1994).

[29] E. Karimi, B. Piccirillo, L. Marrucci, and E. Santamato, Opt. Lett. 34, 1225 (2009).

[30] L. Allen and M. J. Padgett, Optics Communications 184, 67 (2000).

[31] J. Leach, S. Keen, M. J. Padgett, C. Saunter, and G. D. Love, Optics express 14, 11919 (2006).

[32] We defined $\left\langle\mathbf{x}_{\perp}(z)\right\rangle=\int \mathrm{d} x \mathrm{~d} y \mathbf{x}_{\perp}|\Psi(x, y, z)|^{2}$. Due to the paraxial propagation in general we have $\left\langle\mathbf{x}_{\perp}(z)\right\rangle=$ $\left\langle\mathbf{x}_{\perp}(0)\right\rangle+\frac{\left\langle\mathbf{k}_{\perp}\right\rangle}{k} z$, namely the centroid travels in a straight line as the beam propagates: identifying such line with the $z$ axis is equivalent to set $\left\langle\mathbf{x}_{\perp}(z)\right\rangle=0$.

[33] W. H. Carter, Applied Optics 19, 1027 (1980).

[34] We use a different notation with respect to [16, where the beam radius is defined as $\sqrt{2} \sigma_{r}(z)$.

[35] B. E. A. Saleh and M. C. Teich, Fundamentals of Photonics, Wiley (1991).

[36] N. G. De Bruijn, Uncertainty Principles in Fourier Analysis (1967).

[37] N. Hodgson and H. Weber, Laser Resonators and Beam Propagation, volume 108 of Springer Series in Optical Sciences, Springer-Verlag, New York (2005), ISBN 0-38740078-8.

[38] A. E. Siegman, New developments in laser resonators, Proc. SPIE 1224, Optical Resonators, (1990).

[39] E. Karimi, G. Zito, B. Piccirillo, L. Marrucci, and E. Santamato, Opt. Lett. 32, 3053 (2007).

[40] E. Karimi, B. Piccirillo, L. Marrucci, and E. Santamato, Opt. Exp. 16, 867 (2008).

[41] J. A. Nelder and R. Mead, The Computer Journal 7, 308 (1965).

[42] M. A. Alonso, Journal of Optics 13, 064016 (2011).

[43] F. Tricomi, Mathematische Zeitschrift 53, 136 (1950).

[44] J. A. Nelder and R. Mead, The Computer Journal 7, 308 (1965). 